\title{
Expression and regulation of long noncoding RNAs in TLR4 signaling in mouse macrophages
}

\author{
Ai-Ping $\mathrm{Mao}^{1 \dagger}$, Jun Shen ${ }^{2 \dagger}$ and Zhixiang Zuo ${ }^{3^{*}}$
}

\begin{abstract}
Background: Though long non-coding RNAs (IncRNAs) are emerging as critical regulators of immune responses, whether they are involved in LPS-activated TLR4 signaling pathway and how is their expression regulated in mouse macrophages are still unexplored.

Results: By repurposing expression microarray probes, we identified 994 IncRNAs in bone marrow-derived macrophages (BMDMs) and classified them to enhancer-like IncRNAs (elncRNAs) and promoter-associated IncRNAs (plncRNAs) according to chromatin signatures defined by relative levels of H3K4me1 and H3K4me3. Fifteen elncRNAs and 12 plncRNAs are differentially expressed upon LPS stimulation. The expression change of IncRNAs and their neighboring protein-coding genes are significantly correlated. Also, the regulation of both elncRNAs and plncRNAs expression is associated with H3K4me3 and H3K27Ac. Crucially, many identified LPS-regulated IncRNAs, such as IncRNA-Nfkb2 and IncRNA-Rel, locate near to immune response protein-coding genes. The majority of LPS-regulated IncRNAs had at least one binding site among the transcription factors p65, IRF3, JunB and cJun.
\end{abstract}

Conclusions: We established an integrative microarray analysis pipeline for profiling IncRNAs. Also, our results suggest that IncRNAs can be important regulators of LPS-induced innate immune response in BMDMs.

Keywords: TLR4, LPS, elncRNA, plncRNA, Histone modification

\section{Background}

TLR4, a founding member of the TLR family, is a pattern recognition receptor for lipopolysaccharide (LPS) that can induce inflammatory response and cause septic shock [1]. Stimulation of TLR4 by LPS results in the rapid activation of transcription factors, the best characterized of which are interferon regulatory factors (IRFs), the nuclear factorkappa B (NF-kB) and activator protein 1 (AP-1) families.

In recent years, tens of thousands of long non-coding RNAs (lncRNAs) have been identified in the mammalian genomes, many of which have been implicated in a range of developmental processes and diseases [2-5]. Though most of lncRNAs have been primarily studied in the context of genomic imprinting, developmental process and cancer, lncRNAs are now emerging as important regulators of both innate and adaptive immune responses [6]. Mammalian $\mathrm{CD} 11 \mathrm{c}+$ dendritic cells produce many thousands of

\footnotetext{
* Correspondence: zzuo@bsd.uchicago.edu

${ }^{\dagger}$ Equal contributors

${ }^{3}$ Department of Medicine, University of Chicago, 900 East 57th street,

Chicago, IL 60637, USA

Full list of author information is available at the end of the article
}

IncRNAs when stimulated with LPS [7]. The IncRNA Ptpri-as1 is highly expressed in macrophage-enriched tissue and transiently induced by TLR ligands with similar pattern to Ptprj [8]. TLR signaling also induces IncRNACox2, which serve as both repressor and activator of genes through interactions with various regulatory complexes [9]. $\mathrm{Li}$ et al. identified a IncRNA THRIL regulating TNF $\alpha$ expression through its interaction with hnRNPL during innate activation of THP1 macrophages [10]. Using a global clustering algorithm based on ChIP-seq signals of RNA polymerase II and H3K4me3, Garmire et al. identified a list of putative lincRNAs in mouse macrophages [11]. Most recently, Ilott et al. discovered that both canonical lncRNAs and enhancer lncRNAs regulated the LPS-induced inflammatory response in human monocytes [12]. However, systemic characterization of LPS-regulated lncRNAs in mouse BMDMs is lacking so far.

More and more studies have suggested that although lncRNAs are not specifically targeted in the original array design, a large portion of probes can be reannotated for interrogating lncRNA expression [13-19]. Compared to RNA-seq of low sequencing coverage, microarray data 
have lower technical variations and higher sensitivity for transcripts with low abundance [20,21], which is a markedly feature of lncRNAs [3]. Additionally, microarray datasets contain strand information, thus allow for interrogating the expression of antisense lncRNAs.

In this study, we aim to explore the activities and potential functions of lncRNAs in LPS-induced innate immune response in mouse BMDMS. To this end, we firstly repurposed different expression microarray platforms to identify lncRNAs from reannotated probes. We then performed an integrative expression analysis of these identified lncRNAs on publicly available expression datasets on LPS-stimulated BMDMS. By using qRT-PCR, we validated the expression changes of some lncRNAs. We classified the lncRNAs to elncRNAs and plncRNAs according to chromatin status defined by relative levels of H3K4me1 and H3K4me3 surrounding transcription start sites. We further examined the correlation of the expression change between lncRNAs and nearest neighboring protein-coding genes. Crucially, several lncRNAs are near to immune response genes, and these pairs are significantly co-expressed, such as lncRNANfkb2/Nfkb2, lncRNA-Rel/Rel. The majority of LPSregulated lncRNAs have at least one binding site among the transcription factors p65, IRF3, JunB and cJun, further indicating their potential roles in immune response.

\section{Results}

\section{Reannotating microarray probes for IncRNAs in BMDMs}

To systematically identify LPS-regulated lncRNA profile, we utilized publicly available microarray datasets and reannotated the probes using a comprehensive computational pipeline as illustrated in Figure 1A. From 12 published datasets including six different platforms from Affymetrix, Agilent and Illumina (Additional file 1), we identified 3988 lncRNAs (Additional files 2 and 3). We then incorporated evidence of TSS by TSS-seqs such as CAGE [22] and nanoCAGE [23] or epigenetic markers to filter the lncRNAs. We collected all publicly available mouse TSS-seqs to construct a comprehensive database for mouse gene TSS annotations. Based on the TSS database, we discarded the lncRNAs with no TSS-seq supported or ambiguous TSSs overlapping with neighboring protein-coding genes. Furthermore, we utilized publicly available ChIP-seq data (Additional file 4) to examine the epigenetic markers around the IncRNAs' TSS region. Those lncRNAs with any epigenetic modifications of H3K4me1, H3K4me3 and PolII were retained. This resulted in 994 reliable lncRNAs with independent transcription evidence (Additional file 5). Although different platforms differed in the lncRNA compositions, they shared a large number of lncRNAs (Figure 1B). We also reannotated the probes to protein-coding genes for all the platforms for further analysis (Additional file 6).

We classified lncRNAs based on their proximity and relative orientation to protein-coding genes (Figure $1 \mathrm{C}$ ).
The 994 lncRNAs with TSS evidence were classified as follows: exonic sense (overlapping a protein-coding gene exons on the same strand), intronic sense (only overlapping a protein-coding gene introns on the same strand), antisense (overlapping a protein-coding gene locus on the opposite strand), biodirectional (on the opposite strand to a protein-coding gene locus and the distance of TSSs is within $1 \mathrm{~kb}$ ), and intergenic (no-overlapping with a protein-coding gene locus and besides biodirectional) (Figure 1C). The number and distribution of lncRNAs among the different classes were: exonic sense (49, 4.9\%), intronic sense $(28,2.8 \%)$, antisense (402, 40.4\%), bidirectional (224, 22.5\%), intergenic $(291,29.3 \%)$ (Figure 1D; Additional file 7). Since majority of exonic sense lncRNAs may simply represent fragments of $5^{\prime}$ and $3^{\prime}$ UTRs or nonsensemediated mRNA decay (NMD) isoforms of proteincoding genes [24], we excluded exonic sense lncRNAs from further analysis.

\section{LPS-regulated IncRNAs in BMDMs}

Based on the reannotated probes, we obtained LPSregulated lncRNAs in BMDMs from individual datasets, the majority of which have LPS stimulation time points ranging from 3 to 6 hours. We then used Pearson correlation analyses to evaluate the consistency of microarrays within each manufacture and across manufactures. The correlation of all pairs of microarrays is in the range from -0.26 to 0.80 (Figure 2A). With only a few exceptions, majority of the pairs are showing significant positive correlation. The pairs that had low correlation only because there were limited overlaps between them. The expression pattern of lncRNAs in different datasets from the same manufacture had remarkably high correlation (Figure 2A and B). Moreover, the expression of overlapped lncRNAs could also be largely validated by cross-manufacture datasets (Figure $2 \mathrm{~A}$ and $\mathrm{B}$ ). The consistency of lncRNA expression represented by multiple probes from different platforms suggests the reliability of probe reannotation. Though different platforms of LPSregulated lncRNA expression have overall agreements, some varieties also exist. To integrate these microarrays in an unbiased manner, we exploited a recently published robust rank aggregation algorithm [25] (Additional file 8). Different platforms for protein-coding genes had even stronger consistency (Additional file 9), and we also integrated their expression using the same strategy (Additional file 10). To validate the results, we randomly selected 10 LPS-regulated lncRNAs to do quantitative RT-PCR (qRT-PCR) in BMDMs stimulated by different concentrations of LPS for 0,3 and 6 hours (Additional file 11). The fold changes determined by qRT-PCR were strongly correlated with integrated microarray analysis result (Figure 3A, $r=0.86$ ). Using bonferroni-adjusted $P$ value 0.05 as the cutoff, we identified 15 upregulated 


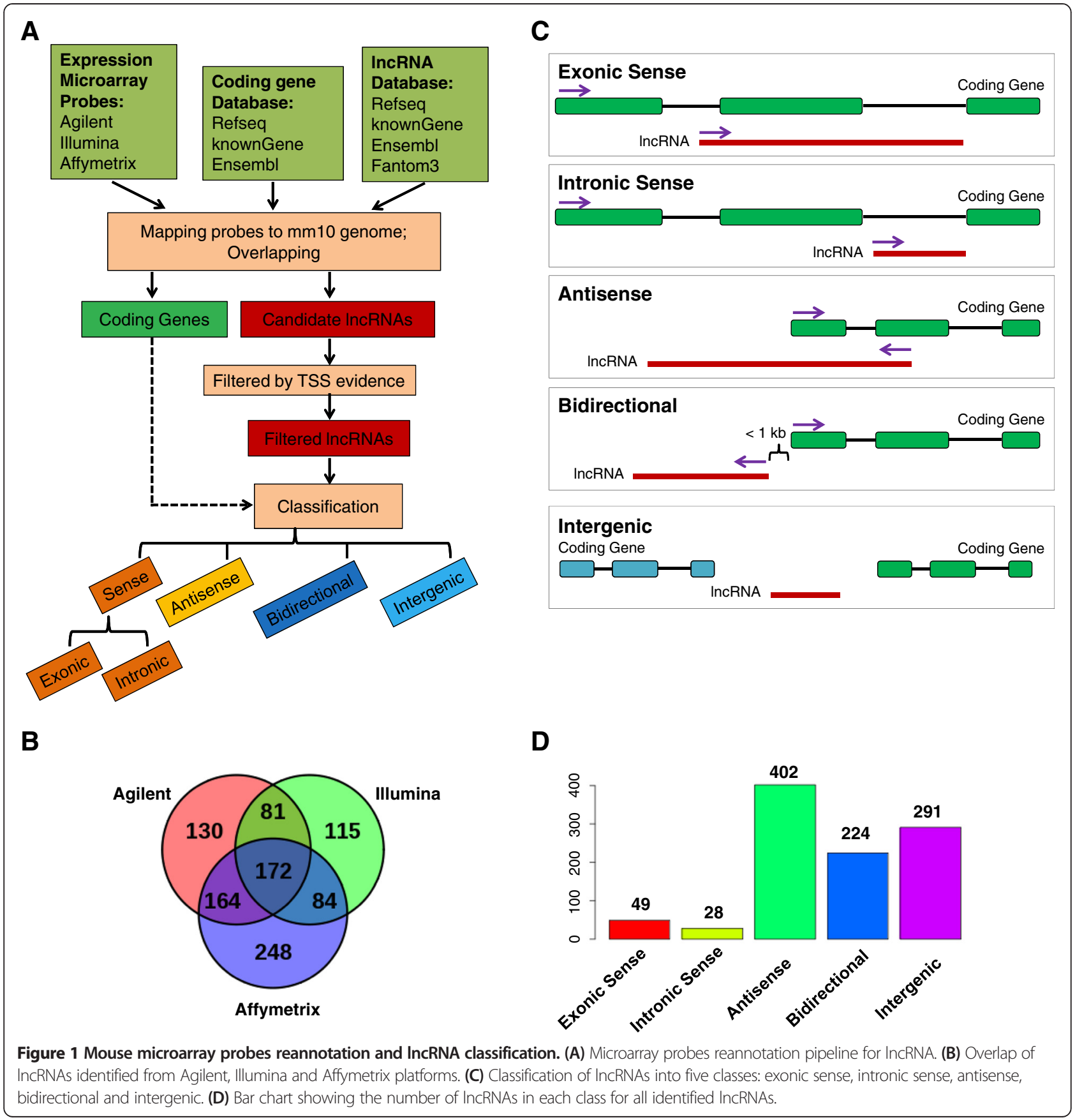

and 12 downregulated lncRNAs upon LPS stimulation in BMDMs (Figure 2B; Additional file 12). Markedly, the expression change of two upregulated and three downregulated lncRNAs significantly stimulated by LPS was confirmed by qRT-PCR (Figure 3B). As expected, the fold changes of lncRNAs showed some dependence on LPS stimulation time and concentration. Taken together, we accurately recapitulated the lncRNA expression changes upon LPS stimulation.
Chromatin signatures separate elncRNAs and plncRNAs

Previous studies have suggested that the ratio of H3K4me1/ H3K4me3 around TSSs can separate lncRNAs into elncRNAs and plncRNAs [26-30]. To classify the lncRNAs identified from microarrays accordingly, we utilized publicly available histone modification ChIP-seq data (Additional file 4). We calculated the relative ratio of H3K4me1/H3K4me3 in a four Kb window centered on TSSs. Of note, $370(37.2 \%)$ of 994 lncRNAs showed 


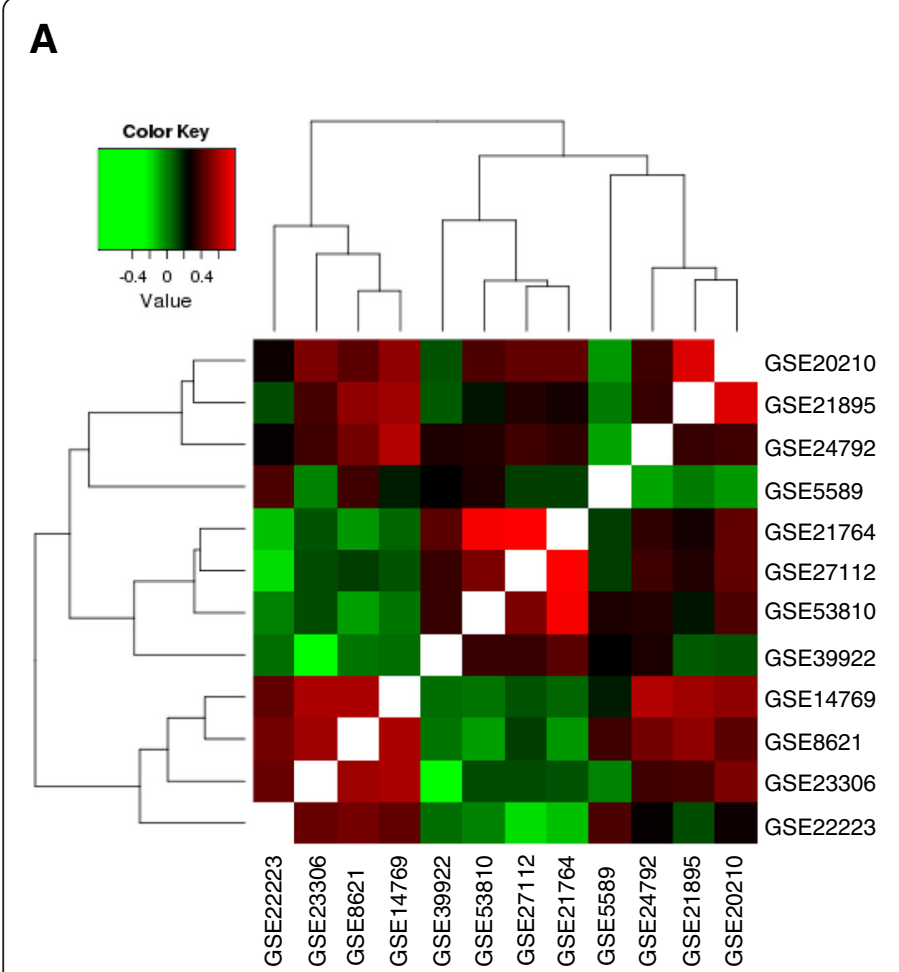

B
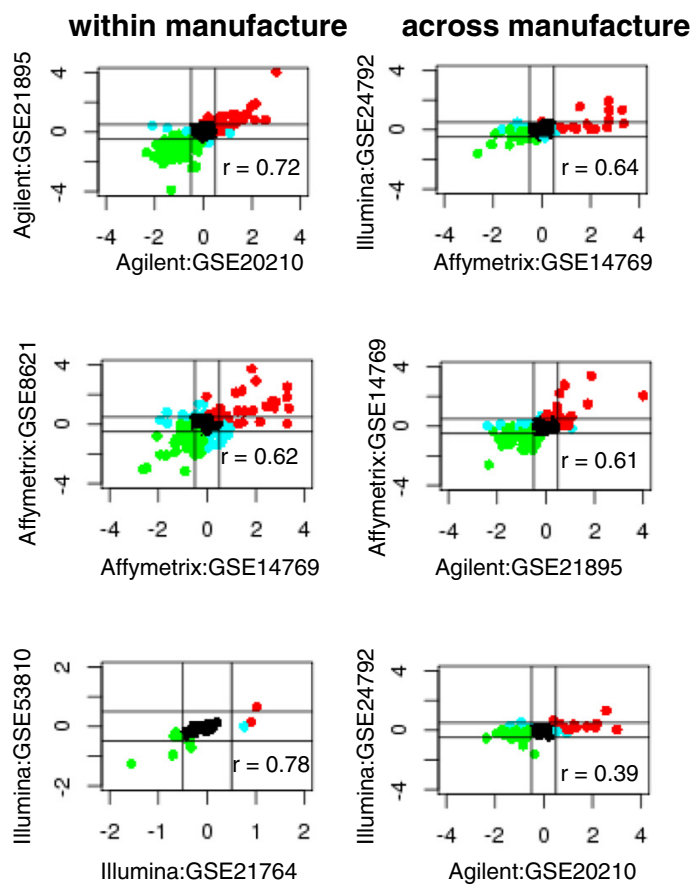

C

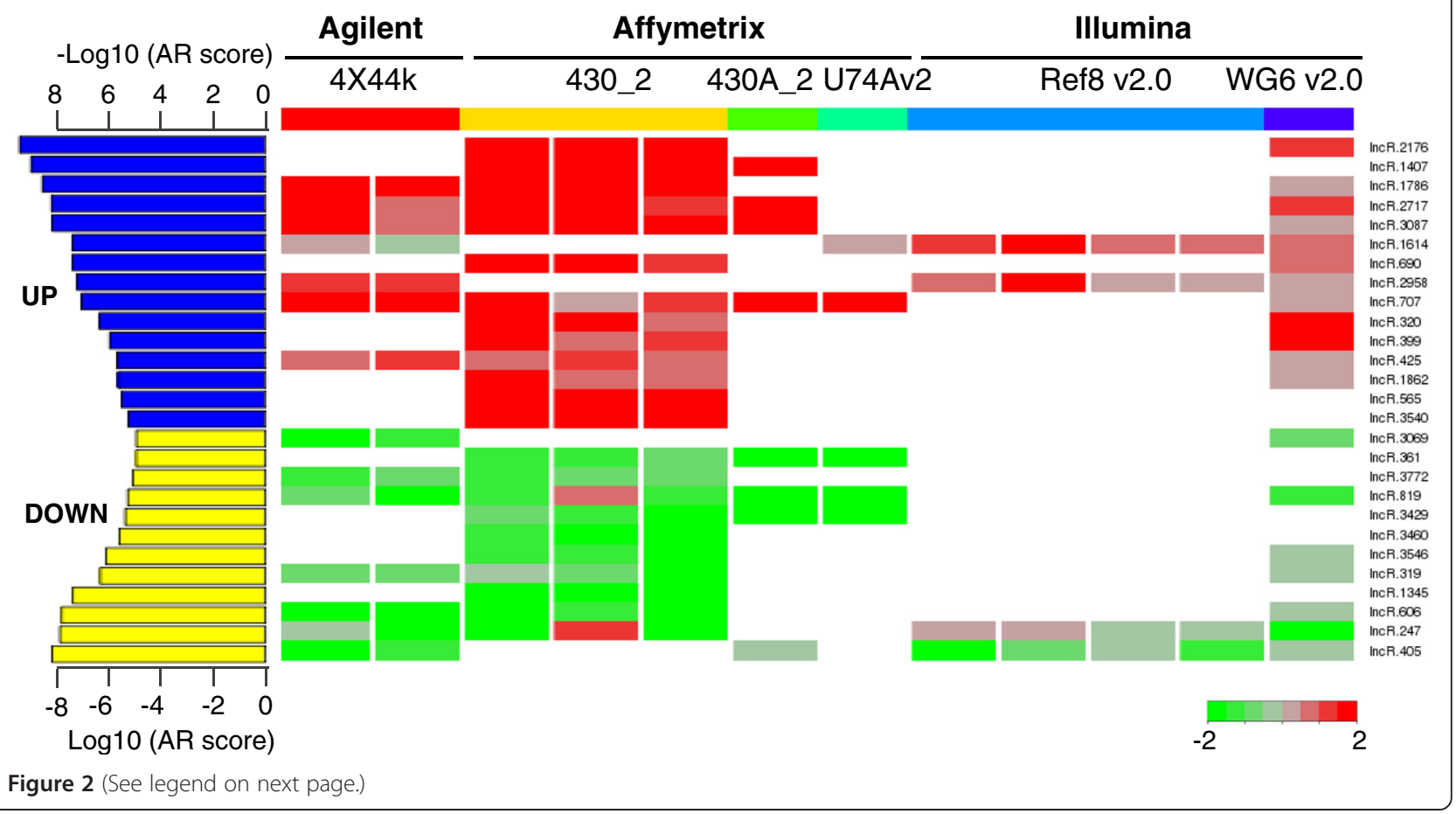


(See figure on previous page.)

Figure 2 Correlation between different microarray datasets and integratation of LPS-regulated IncRNAs from all the datasets.

(A) Heatmap showing the Pearson correlation value between all the possible pairs of microarray datasets. (B) Represented correlation of the log 2 expression change of LPS-regulated IncRNAs within (left panel) and across manufactures (right panel). (C) The right panel is the heatmap of Log2 expression change of 27 significantly changed IncRNAs upon LPS-stimulation. The fold change was scaled to -2 to 2 by setting all values more than 2 or less than -2 to 2 and -2 , respectively. The IncRNAs list was defined by Bonferroni-adjusted p value cutoff 0.05 , which was calculated from a robust rank aggregation (RRA) algorithm. On the left panel, the aggregation rank score (AR score) from RRA was shown. AR score indicated the integrated rank from the integrated analysis of fold change from 12 different microarray datasets. For upregulated IncRNAs, -Log10(AR score) was used while for downregulated IncRNAs, Log10(AR score) was used.

enhancer-like features (H3K4me1/H3K4me3 high), while the remaining lncRNAs displayed promoter histone signatures (H3K4me1/H3K4me3 low) (Figure 4A; Additional file 13). LPS stimulation had marginal effect on the relative ratio of H3K4me1/H3K4me3, thus lncRNAs displayed similar enhancer-like or promoter-associated histone signatures in both unstimulated and LPS stimulated BMDMs
(Figure 4A). As expected, both elncRNAs and plncRNAs were enriched with H3K27Ac modification, a well-accepted mark of biological activity (Figure 4A). These LPS-regulated plncRNAs and elncRNAs can be either induced or repressed by LPS stimulation, and no difference was detected between plncRNAs and elncRNAs (Figure 4B, p=0.83, chi-square test).

\section{A}

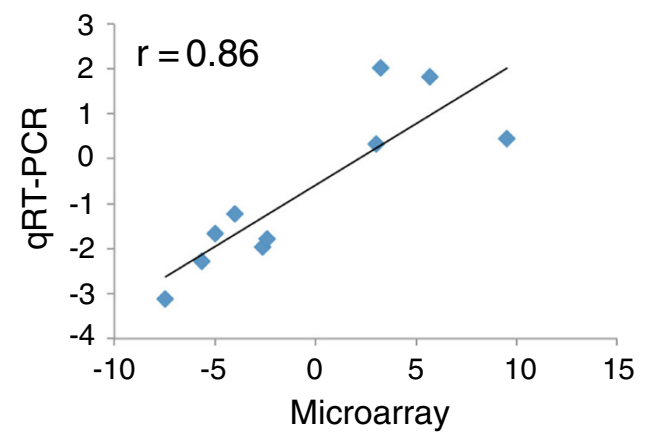

B

IncR.2176 (NR_027919; uc008mau.2)

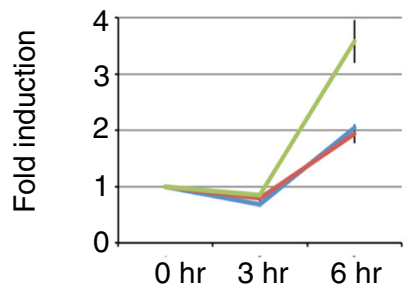

IncR.1345

(2810001A02)

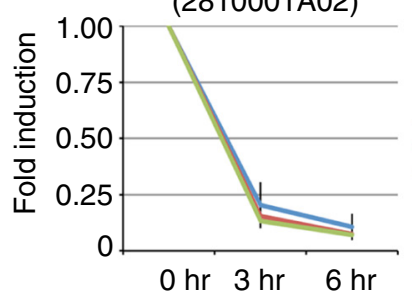

IncR.1862

(F830019M12; 4833438C02)

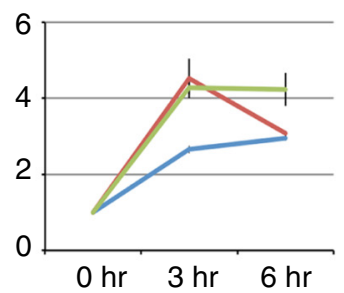

IncR.3460

(NR_037959;uc009jer.1; 9830164F18)

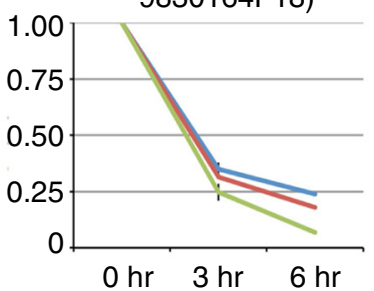

$$
\begin{aligned}
& -10 \mathrm{ng} / \mathrm{ml} \\
& -100 \mathrm{ng} / \mathrm{ml} \\
& -1 \mathrm{ug} / \mathrm{ml}
\end{aligned}
$$

Figure 3 qRT-PCR validations of LPS-regulated IncRNAs from integrated microarray datasets. (A) Correlation analysis of averaged log 2 (fold change) derived from qRT-PCR and $+/-\log _{10}$ (AR score) derived from integrated microarrays as shown in Figure 2C. (B) The relative expression levels of two upregulated and three downgregulated IncRNAs identified by integrated analysis in BMDMs stimulated with $10 \mathrm{ng} / \mathrm{ml}, 100 \mathrm{ng} / \mathrm{ml}$ and 1 $\mathrm{ug} / \mathrm{ml} \mathrm{LPS}$ for 0,3 and 6 hours. 
A

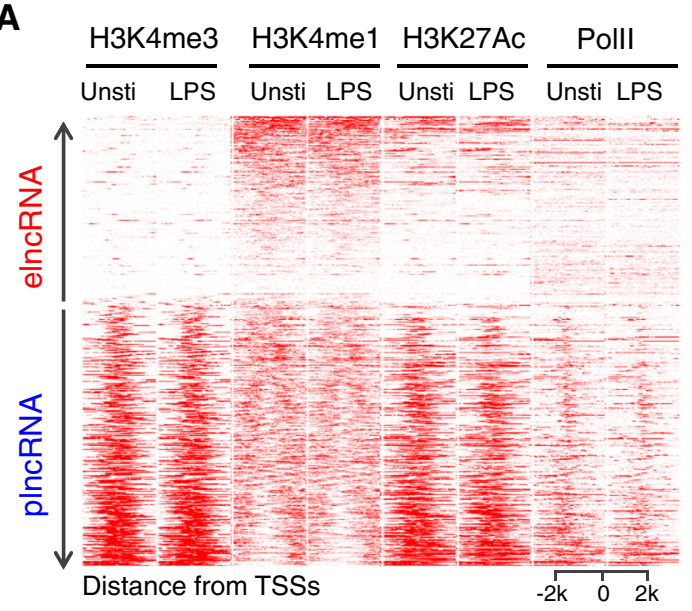

B

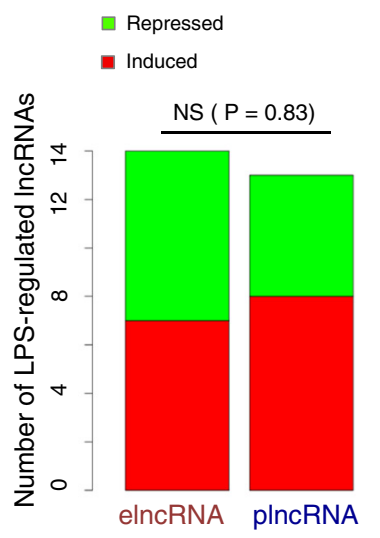

C
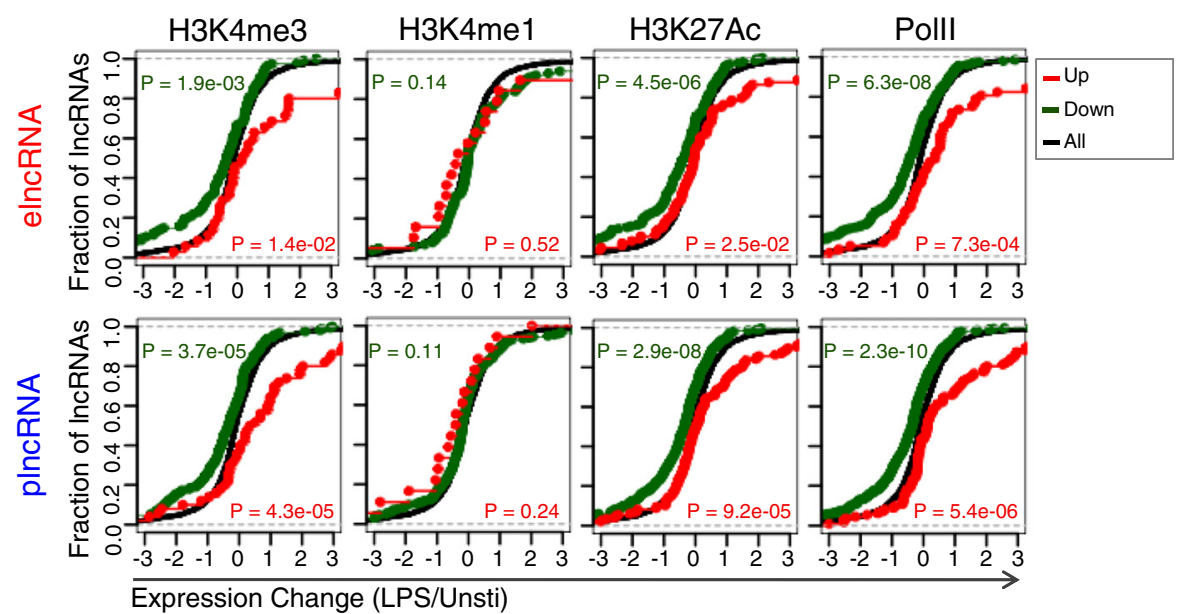

Figure 4 Chromatin signatures surrounding TSSs separate IncRNAs into elncRNAs and plncRNAs. (A) Heatmap presenting the read intensities of H3K4me3, H3K4me1, H3K27Ac and Polll modification across a 4 Kb interval centered on TSSs of the 994 IncRNAs before and after LPS stimulation in BMDMs (the $y$-axis indicates 994 IncRNAs and the label was not shown, refer to Additional file 13). (B) The numbers of LPSupregulated and -downregulated IncRNAs among elncRNAs and plncRNAs showed by bar chart. The P value was calculated by chi-square test. (C) Empirical cumulative distribution function (ecdf) plot was showing to indicate the correlation between the change epigenetic markers (H3K4me3, H3K4me1, H3K27Ac and PollI) and the expression change of their corresponding IncRNAs. Upper panel is elncRNAs, while lower panel is plncRNAs. The red and blue curves represent IncRNAs that were marked with increased and decreased epigenetic markers, respectively. Black curve represent all IncRNAs. Based on the knowledge that all these four epigenetics marks are active markers that positively correlated with expression changes, one-sided KS-test was performed to evaluate the difference between red curve and black curve, and similarly the difference between blue curve and black curve was evaluated.

It has been well established that histone modification changes are associated with changes of IncRNA expression, which is confirmed in our finding. We observed that differences in H3K4me3 and H3K27Ac were positively correlated with changes in both elncRNAs and plncRNAs expression (Figure 4C). H3K4me1 was not associated with expression change upon LPS stimulation for both elncRNAs and plncRNAs (Figure 4C). PolII signal for LPS regulated elncRNAs and plncRNAs was significantly changed upon LPS stimulation (Figure 4C).

\section{Correlation between IncRNA and neighboring protein- coding gene expression}

LncRNAs have been reported to coordinate the regulation of neighboring protein-coding genes through a locus control process [31]. We assessed their relative 
distance and correlation of expression changes to neighboring gene for elncRNAs and plncRNAs, respectively. Interestingly, when considering intergenic and bidirectional lncRNAs only, although not significant, elncRNAs were in a closer proximity to protein-coding genes than plncRNAs (Figure $5 \mathrm{~A}, \mathrm{p}=0.066, \mathrm{t}$ test). The elncRNAs and plncRNAs had similar compositions of lncRNA classes (Figure 5B, $\mathrm{p}$ value $=0.93$, chi-square test). Although the distance to nearest protein-coding genes for plncRNAs is not as close as elncRNAs, all the intronic sense, antisense, bidirectional and intergenic elncRNAs/plncRNAs are significantly co-expressed with neighboring protein-coding genes (Figure $5 \mathrm{C}$, all $\mathrm{p}<0.05$, KS test). Intronic sense lncRNA expression change has the strongest correlation with protein-coding gene neighbors, compared to other three classes (Figure 5C), suggesting that intronic sense
lncRNAs are probably often co-transcribed with closest mRNA genes.

\section{LPS-regulated IncRNAs closely related to inflammatory response}

We found 24 out of 27 LPS-regulated lncRNAs were adjacent to or overlapped with protein-coding genes. Adapting the previously proposed nomenclature [32], we renamed the 24 lncRNA genes based on their neighboring proteincoding genes (Additional file 14). Notably, the majority of these IncRNAs (19/24) were positively correlated with neighboring protein-coding genes, while only 5 of 24 were negatively correlated (Figure 6A). Of particular interest was LPS-regulated lncRNAs paired with neighboring protein-coding genes known to play roles in inflammatory response, such as NFKB pathway genes Nfkb2 and Rel

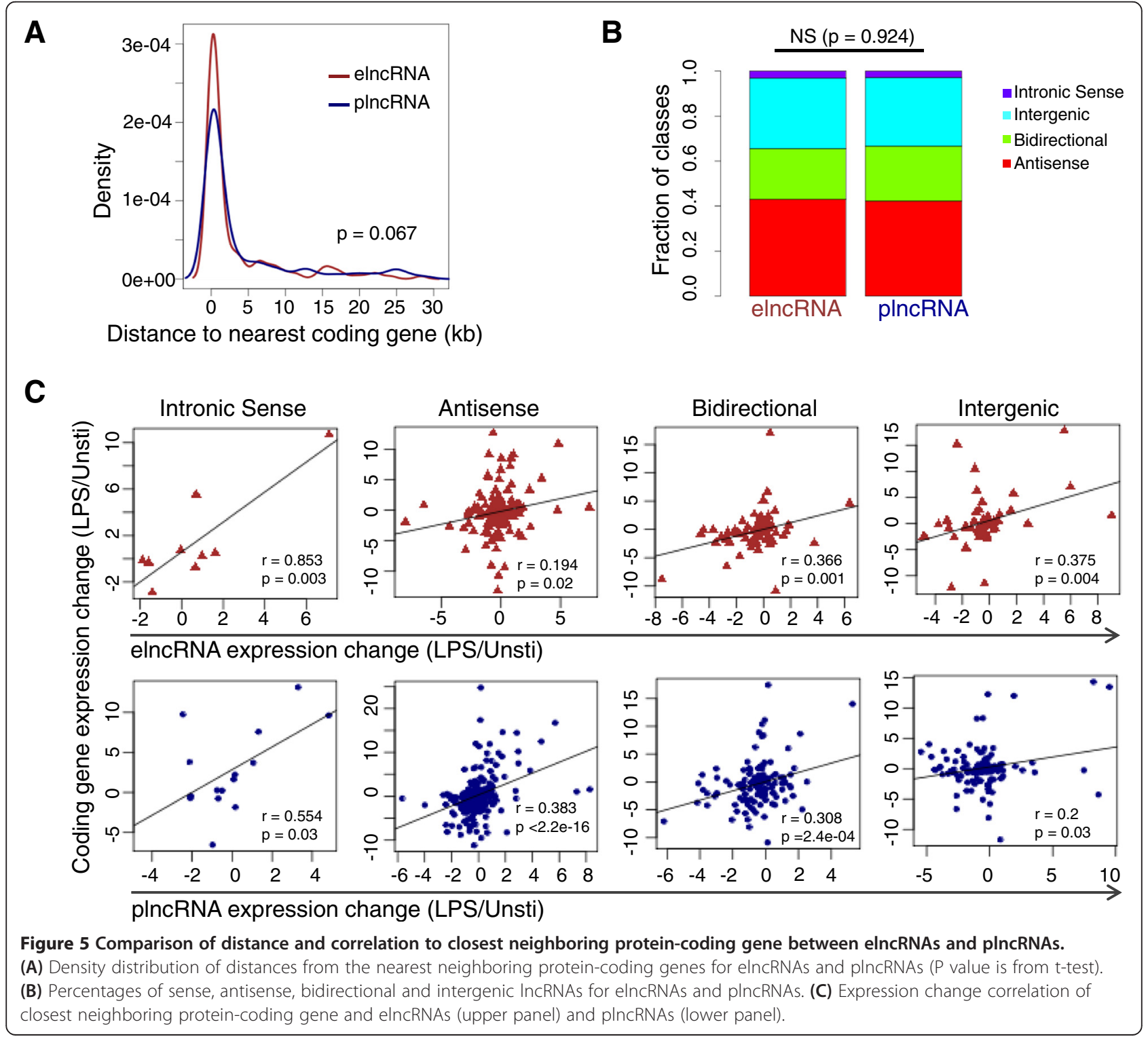


A

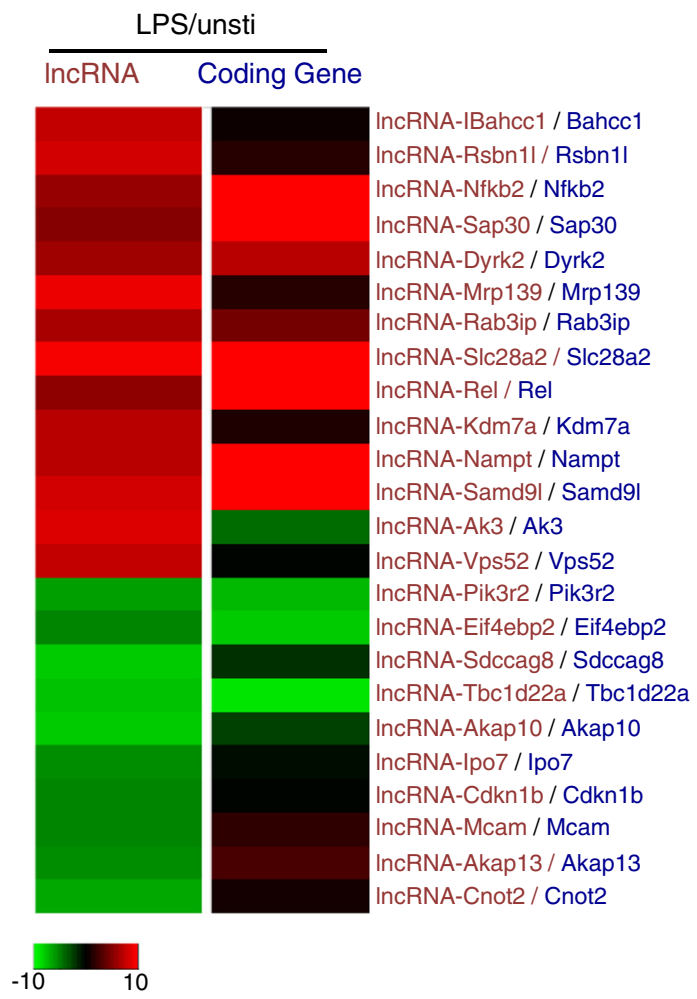

B

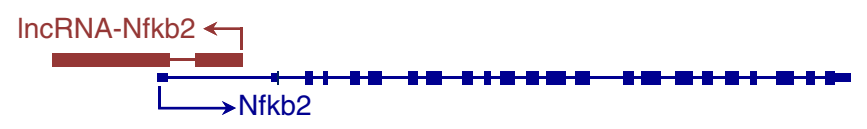

IncRNA-Nfkb2 (IncR.1862 / F830019M12; 4833438C02)
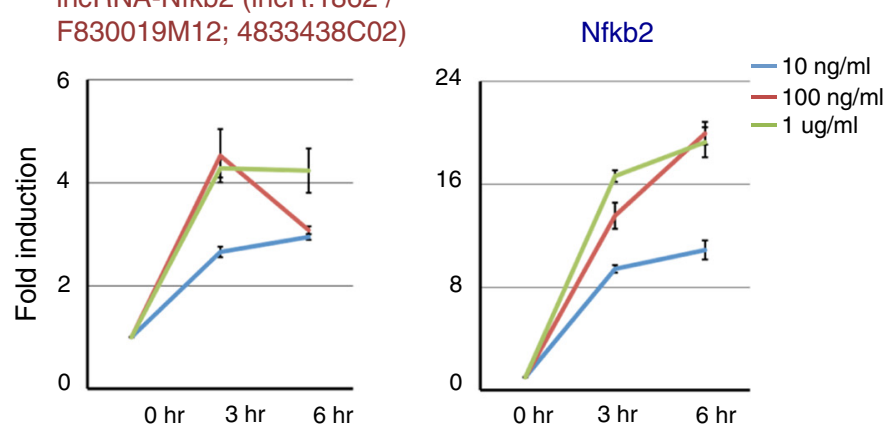

Figure 6 Co-regulation between IncRNA and neighboring protein-coding gene expression upon LPS stimulation in BMDMs.

(A) Heatmap showing the integrated expression change of LPS-regulated IncRNAs and nearest protein-coding gene neighbors. (B) qRT-PCR validation of three InCRNA-protein-coding gene pairs co-regulated upon LPS stimulation in BMDMS.

(Figure 6A). Using qRT-PCR, we validated the co-expressed lncRNA-Nfkb2 and NFkb2 pairs in BMDMs stimulated with various concentrations of LPS for 0,3 and 6 hours (Figure 6B).

\section{LPS-induced transcriptional regulation of IncRNAs in BMDMs}

In resting BMDMs, the transcription factors p65, IRF3 and AP-1 family members JunB and cJun are sequestered in cytoplasm. Upon LPS stimulation, these transcription factors are rapidly translocated to nucleus, where they act alone or together with one another to bind numerous gene loci to regulate gene expression. To determine whether these transcription factors were required for transcriptional regulation of lncRNAs, we took advantage of published ChIP-seq data and reanalyzed the peaks. Firstly, we found that both LPS-regulated elncRNAs and plncRNAs were enriched for binding sites of the four transcription factors. Interestingly, the transcription factors binding sites were enriched in lncRNAs whose expression was either increased or decreased after LPS stimulation, for both elncRNAs and plncRNAs (Figure 7A). Bcl6 is a transcriptional factor that binds genes and broadly constrains the inflammatory response through cistromic antagonism of a
TLR-NF- $\mathrm{kB}$ network [33]. We detected the similar enrichment pattern for Bcl6 before and after LPS stimulation.

Of the 27 LPS-regulated elncRNAs and plncRNAs identified in BMDMs, 23 were bound by at least one of the four transcription factors (p65, IRF3, JunB and cJun) (Figure 7B). p65, IRF3, JunB and cJun bound to 13, 20, 19 and 9 lncRNAs in BMDMs exposed to LPS, respectively (Figure 7B). Of note, 5 lncRNAs were bound by all the four transcription factors. Different transcription factor binding sites of lncRNAs were within average $2 \mathrm{~Kb}$, suggesting that these proteins acted together to regulated lncRNA expression. Interestingly, the majority of Bcl6 binding sites (80\% $(4 / 5)$ and $100 \%$ (1/1) in LPS-unstimulated and -stimulated BMDMs, respectively) colocalized with at least one of IRF3, p65, JunB and cJun sites after LPS stimulation (Figure 7C). Above findings indicated that IRF3, p65 and AP-1 family member JunB and cJun were the major transcription factors that acted in a synergetic manner and regulated lncRNAs expression in TLR4 signaling pathway, while Bcl6 antagonized some lncRNA binding sites to prevent hyperimmune response. For example, lncRNA-Ipo7 was co-bound by IRF3 and JunB within a small window (Figure 8A). Out of two potential promoter regions for lncRNA-rel, one was bound by p65, IRF3, JunB 


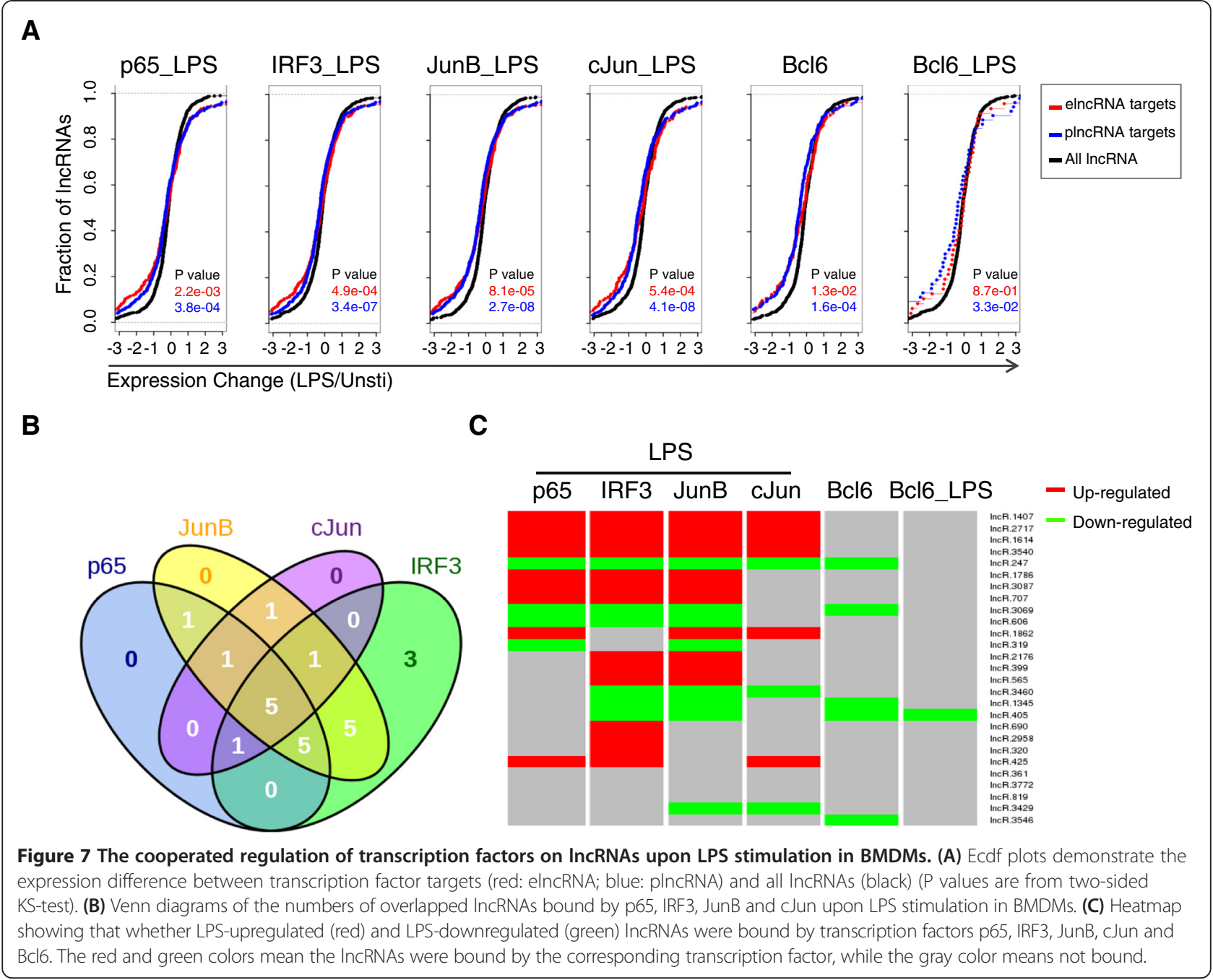

and cJun in BMDMs after LPS stimulation, and the other one was bound by IRF3 and JunB after LPS stimulation, while the binding site was antagonized by Bcl6 under rest condition (Figure 8B).

\section{Discussion}

Previous studies mainly focused on the study of LPSregulated protein-coding genes but ignored the function of lncRNAs involved. To explore the potential role of lncRNAs in the activation of TLR4 signaling, we constructed a comprehensive bioinformatics pipeline to reannoate probes to lncRNA from literature expression microarray datasets in BMDMs. Giving that large number of such datasets are available in public repositories, the pipelines we generated will be useful for reannotating array probes to address different biological questions.

Our integrated lncRNA and protein-coding gene expression profiles are valuable resources for understanding the LPS-stimulated program, as well as their co-regulation. Having established that LPS induced widespread changes in the expression of lncRNAs in mouse macrophages situated close to differentially expressed immune response-related genes, it was important to determine whether these were functionally relevant. Of great interest was the identification of differentially expressed lncRNAs that are located close to two members of Ref/Nfkb family, Nfkb2 and Ref, which are classical proinflammatory transcription factors known to play critical roles in both innate and adaptive immune response. Nfkb2 was reported to be upregulated upon LPS stimulation in human monocytes [34]. Our qRT-PCR experiments confirmed the co-expression of lncRNA-Nfkb2 and Nkfb2. It is unlikely that the co-regulation of lncRNAs and Ref/Nfkb family was a random phenomenon since two members of this family were found to be co-regulated with lncRNAs. Given the importance of Ref/Nfkb family in immune response, a further examination of the function and mechanism for their co-located and co-expressed lncRNAs is worth doing. However, our study has limitations to detect known LPS-regulated lncRNAs due to the lack of probes. Previous study indicated that Cox 2 and lncRNA-Cox 2 were 

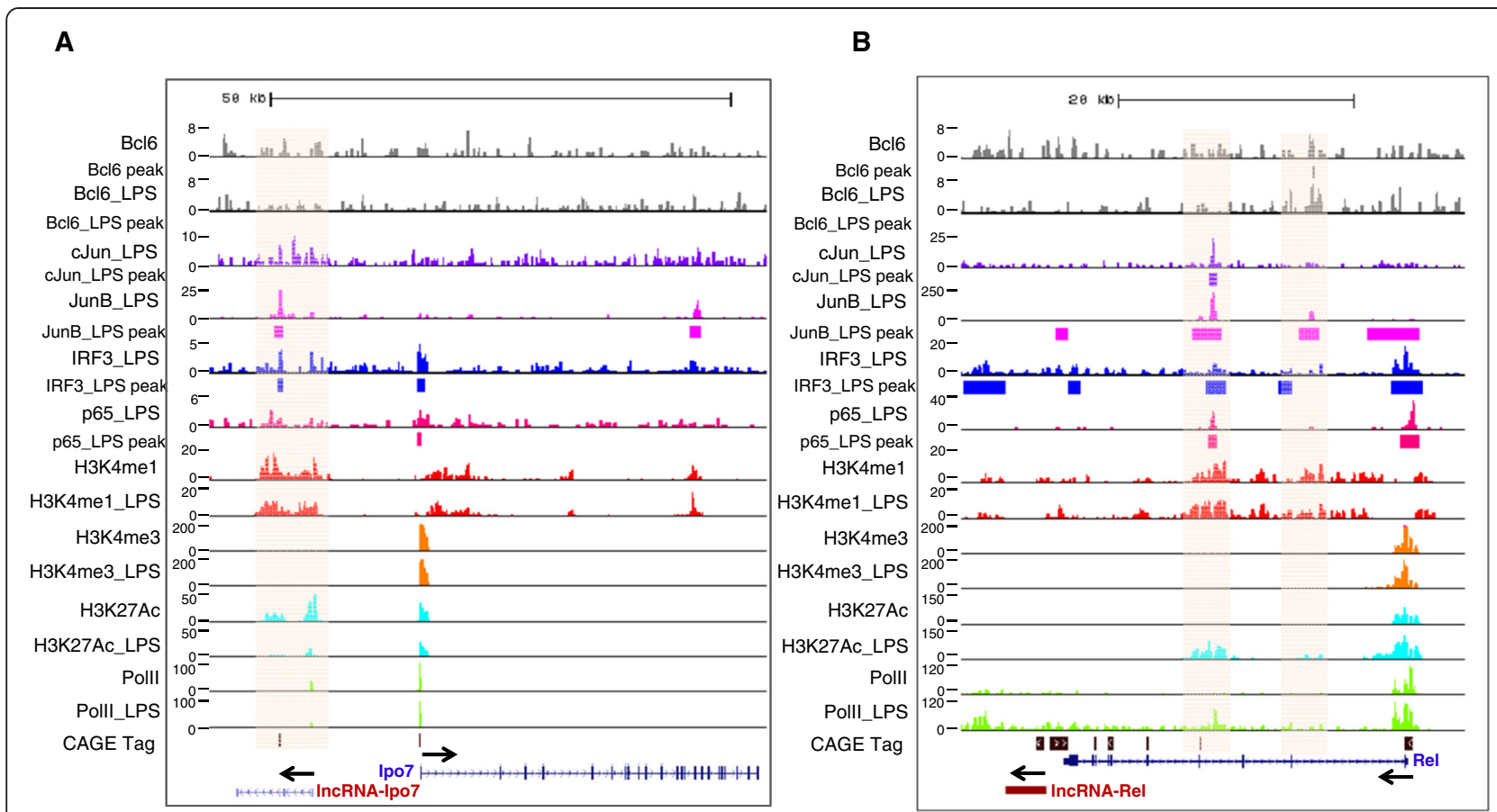

Figure 8 The transcription factors binding and histone modifications. (A) IncRNA-lpo7 and Ipo7. (B) IncRNA-Rel and Rel. Arrows indicate the direction of transcription.

markedly induced after TLR4 stimulation in BMDMs [9]. Due to the lack of probes for lncRNA-Cox2, we did not detect IncRNA-Cox2 in this study. We performed qRT-PCR to confirm the co-expression of Cox2 and lncRNA-Cox2 (Additional file 15). It should be noted that we applied a stringent strategy to derive a confident list of LPS-regulated lncRNAs. Some interesting lncRNAs are also filtered out, such as IncRNA-Lyn-intron1. Previous study has demonstrated that lncRNA-Lyn spans the first exon and first intron region of Lyn and the expression is increased along with Lyn upon LPS stimulation in BMDMs [9]. We identify a new Lyn associated lncRNA, lncRNA-Lyn-Intron1 (lncR.2430; Additional file 3), located at the first intron of Lyn and $25 \mathrm{~Kb}$ away from lncRNA-Lyn, is also upregulated (Additional file 15). This lncRNA was filtered out because of no clear TSS evidence.

Recent investigation in erythroid cells has suggested that the IncRNAs transcripts are almost evenly divided between elncRNAs and plncRNAs differentiated by chromatin signatures of H3K4me3 and H3K4me1 surrounding transcription start sites [27]. Consistent with this observation, we found that BMDMs expressed elncRNAs and plncRNAs were also evenly distributed. A number of elncRNAs and plncRNAs can be regulated by LPS stimulation. Nevertheless, plncRNAs are more inclined to downregulation upon LPS stimulation compared to elncRNAs. Several previous studies suggested that IncRNA expression changes are regulated by epigenetic mechanism including histone modifications such that H3K27ac, H3K4me3 and H3K36me3 are related to enhancer activity [15]. Similarly, we demonstrated that histone modifications also play important roles in the regulation of IncRNAs upon LPS stimulation in BMDMs. We found that H3K4me3 and H3K27Ac are associated with directionally consistent changes in not only elncRNAs, but also plncRNAs expression. Our studies demonstrate that although distance to nearest neighboring is much nearer in elncRNAs compared to plncRNAs, these both of two kinds of lncRNAs significantly co-expressed with neighboring protein-coding genes. Bidirectional transcription has been shown to be a defining feature of a subset of active enhancers in mouse cortical neurons and human fetal lung fibroblasts $[35,36]$. We have shown that the transcription of bidirectional plncRNAs, as well as elncRNAs, were LPS-stimulation dependent in mouse BMDMs.

The gene program stimulated in TLR4 signaling pathway requires the coordinative activation of transcription factors, of which the most well characterized are p65, IRF3, and AP-1 family members JunB and cJun. Here we demonstrate for the first time that these transcription factors also bind to lncRNAs and regulate their expression upon LPS stimulation. Also, the regulation does not differ between elncRNAs and plncRNAs. The majority of LPS-regulated IncRNAs are bound with at least one of these transcription factors. Markedly, we also identify the up-regulated and down-regulated lncRNAs that are bound by all the four transcription factors, suggesting the widely cooperation of these transcription factors. Recent study suggests that Bcl6 
antagonizes p65 bindings under rest condition to prevent the hyper-activation of inflammatory genes [33]. Interestingly, we found that Bcl6 also binds to a portion of lncRNAs and the binding sites can overlap with not only p65, but also IRF3, JunB and cJun. We speculate these transcription factors may regulate lncRNAs in a similar manner to protein-coding genes upon LPS stimulation in BMDMs.

\section{Conclusions}

Taken together, we have provided a valuable resource of LPS-regulated lncRNA expression profile, together with many potential co-regulated candidate protein-coding genes. Among them, we have identified lncRNAs such as lncRNA-Nfkb2 and lncRNA-Rel that are upregulated along with their corresponding protein-coding genes, which are crucial genes in immune response. Although the mechanisms are currently unknown, we speculate that many of the identified elncRNAs and plncRNAs are important participants of LPS-stimulated innate immune response. We also established an integrative microarray analysis pipeline, which opens new avenues for repurposing published genomic data to study the functions and mechanisms of lncRNAs in interested biology fields.

\section{Methods}

\section{Re-annotation of array probes}

The mouse gene annotations were collected from four sources: NCBI RefSeq [37], UCSC knownGene [38], FANTOM3 [39] and Ensembl [40]. For NCBI RefSeq, the mm10 version of mouse refGene was downloaded, and transcripts beginning with "NR" were treated as non-coding RNAs, while transcripts beginning with "NM" were treated as coding RNAs. For UCSC knownGene, the mm10 version was downloaded and transcripts annotated with "noncoding" were considered as non-coding RNAs, while transcripts annotated with "coding" were considered as coding RNAs. A stringent set of FANTOM3 non-coding RNAs was selected based on the conservation and noncoding votes. The fasta sequences of the stringent FANTOM3 non-coding RNAs were aligned against mm10 genome using blat [41] to obtain mm10 annotation of FANTOM3 non-coding RNAs. For Ensembl, the release 77 for mouse was downloaded, and the transcripts annotated with "protein_coding" were treated as coding RNAs, otherwise as non-coding RNAs. We excluded non-coding RNAs with length $<200$ nt from the four sources, and defined others as long non-coding RNAs (lncRNAs). We reannotated probes of six different platforms from Affymetrix, Agilent and Illumina arrays (Additional file 1: Table S1) for lncRNAs using the following procedure. Firstly, the bed format annotations of all array probes were generated. The mm10 bed files for Affymetrix arrays were directly downloaded from the Affymetrix website (http:// www.affymetrix.com). For Agilent and Illumina arrays, we obtained the probe sequences from the Agilent website (http://www.agilent.com) and NCBI GEO database (http:// www.ncbi.nlm.nih.gov/gds), respectively. The probe sequences were mapped against $\mathrm{mm} 10$ genome using blat, and the bed format annotations of the best hits were generated. Secondly, the bed format annotations of probes were intersected with lncRNA annotations and coding gene annotations to obtain lncRNA probes and coding gene probes, respectively. BedTools [42] were utilized to achieve this end. To avoid hybridizations, the probes that were mapped to multiple lncRNA annotations or coding gene annotations were removed. The summary information of probe reannotation result for each array platform is shown in Additional file 2. As a result, 3988 unique lncRNAs were obtained. The detailed reannotations of all probes of the six platforms for lncRNAs are shown in Additional file 3. The detailed reannotations of all probes of the six platforms for coding genes are shown in Additional file 6.

\section{Determination of transcriptional start sites (TSSs)}

We used CAGE [22] and nanoCAGE [23] TSS-seq to determine genome-wide TSSs for mouse genome as described elsewhere [27]. To obtain full annotation of TSSs for mouse genome, we collected all the available TSS-seq from DBTSS [43] and NCBI SRA [44]. The $\mathrm{mm} 9$ bed files of TSS-Seq sequences were downloaded from DBTSS (ftp://ftp.hgc.jp/pub/hgc/db/dbtss/dbtss_ver8), and then were converted from $\mathrm{mm} 9$ to $\mathrm{mm} 10$ using the UCSC liftOver tools (http://genome.ucsc.edu/cgi-bin/hgLiftOver). The fastq files of mouse TSS-Seq sequences (GSE49459 and GSE39849) were downloaded from NCBI SRA using SRA toolkit. Then the TSS-seq sequences were mapped to mm10 genome using bwa [45]. A perl script was written to integrate all the TSS-Seq to obtain the TSS regions. Briefly, the $5^{\prime}$ end position of each TSS-Seq read was extracted as TSS. TSSs closer than $20 \mathrm{bp}$ and derived from the same strand were clustered. Clusters within $400 \mathrm{bp}$ of each other and on the same strand were further grouped as a TSS region. The TSS regions with less than 20 tags supported were discarded, thus 160116 TSS regions were retained.

\section{Filter IncRNAs by TSS evidence}

We associated the IncRNAs reannotated from arrays to the 160116 TSS regions using BEDTools [42]. The IncRNA region plus $30 \mathrm{~Kb}$ upstream/downstream regions were used to scan for the TSS regions. As a result, 25100 TSS regions were found to locate nearby the 3575 IncRNAs that have determined TSSs. ChIP-seq raw reads for H3K4me3, H3K4me1 and H3K27Ac histone modifications and RNA PolII in unstimulated/LPS BMDMs were downloaded from NCBI GEO database (http://www.ncbi.nlm.nih.gov/gds/) (Additional file 4). The raw reads were aligned to $\mathrm{mm} 10$ mouse genome using bowtie 1.0.1 [46] with the $-\mathrm{m}$ reporting option 
set to 2. The peaks of histone modifications and PolII were called using MACS [47] following published parameters [48]. To further refine the TSS regions for lncRNAs, we integrated chromatin signatures such as histone modifications and PolII occupancy nearby lncRNAs to obtain a list of active and reliable TSS regions. The candidate TSS regions for lncRNAs were examined for the peaks of H3K4me3, H3K4me1 and PolII. Only those TSS regions with at least one peak of them were retained, which resulted in 7474 TSS regions associated with 2629 lncRNAs. To further refine the TSS regions for the lncRNAs, we excluded the ambiguous TSS regions which may be overlapped with the neighboring coding gene TSS regions, resulting in 1503 TSS regions for 994 lncRNAs, as listed in Additional file 5.

\section{IncRNA classification}

The lncRNA classification method was adopted from a previous study [32]. The lncRNAs were classified according to their relation with neighbor coding genes. The neighbor coding genes of IncRNAs were selected on the basis of either the nearest distance to the lncRNA or the longest overlapping regions. The lncRNAs with distance to their neighbor coding gens shorter than $1 \mathrm{~kb}$ and with different orientation as their neighbor coding genes were categorized as bidirectional. The lncRNAs that have not any overlap with the neighbor coding genes and not belong to bidirectional were categorized as intergenic. The lncRNAs overlapping with their neighbor coding genes were categorized as genic. The genic lncRNAs where were further classified as sense or antisense according to the orientation relation with neighbor coding genes. The classification of all lncRNAs in this study is shown in Additional file 7.

\section{Expression analysis of LPS stimulated BMDM}

We collected expression array datasets from the studies on the investigation of transcriptional profile in mouse BMDMs, which resulted in 12 arrays from six array platforms (Additional file 1). The expression datasets were downloaded from NCBI GEO repository [49]. For the Affymetrix and Illumina array datasets, we downloaded the probe-level preprocessed expression matrix file directly. For the Agilent array datasets, we downloaded the raw data, and used the R package "Agi4x44Preprocess" [50] to preprocess the raw data. KNN method [51] was used to fill the missing values for each preprocessed expression matrix. Then the probe-level expression matrix was transformed to gene/lncRNA level using the reannotated information as shown in Additional files $3,4,5$ and 6 . The average expression was taken if multiple probes were mapped to the same transcript. For each transcript, the average expression for unstimulated group and LPS stimulated group were calculated separately.
Then the $\log 2$ fold change between the two groups for each transcript was calculated. The expression profiles in the 12 studies for all the lncRNAs are shown in Additional file 8. The expression profiles in the 12 studies for all the coding genes are shown in Additional file 10. We employed a recently published robust rank aggregation algorithm [25] to integrate these 12 expression profiles in an unbiased manner. A P value was obtained for each transcript to represent the upregulation and downregulation under LPS stimulation, respectively. The bonferroni-adjusted $\mathrm{p}$ value cutoff 0.05 was used to select the significantly changed lncRNAs/ coding genes.

\section{Preparation of BMDMs}

Bone marrow cells were harvested from the femurs and tibias of 8-week-old C57BL/6 mice. BMDMs were generated by culture of bone marrow cells in RPMI medium containing $10 \%$ of FBS and $10 \mathrm{ng} / \mathrm{ml}$ of recombinant M-CSF (R\&D Systems, cat. no. 416-ML-010) for 7 days. Differentiated BMDMs were then stimulated with different concentrations of LPS (Sigma, cat. no. L3024) for 0, 3 and 6 hours in RPMI medium. All the mice were raised in a specific pathogen-free environment at the University of Chicago, and experiments were performed in accordance with the guidelines of the Institutional Animal Care and Use Committee.

\section{RNA isolation and qRT-PCR}

Total RNA from different time points of LPS stimulated BMDM was prepared with TRIZOL (Invitrogen, cat. no. 15596026), according to the manufacture's instruction. The cDNA was synthesized from total RNA using SuperScript First-Strand Synthesis System (Invitrogen, cat. no. 11904018). Q-PCR was performed using SYBR Advantage Premix (Clontech, cat. no. 639676) in Strategene Mx3500 thermocycler. The corresponding primers were listed in Additional file 16.

\section{Determining chromatin signatures at IncRNA TSSs}

We did a quantity assessment for the enrichment of H3K4me3, H3K4me1, H3K27Ac and PolII around each lncRNA TSS region using in-house $\mathrm{R}$ script utilizing Rsamtools [52] in R. The relative enrichment of H3K4me3 and H3K4me1 surrounding the transcription start sites of the lncRNAs ( -2 to $2 \mathrm{~Kb}$ ) was calculated to define elncRNA and plncRNA as previously described [27]. Heatmaps of the elncRNA and plncRNA histone modification profiles were generated using heatmap. 2 function in $\mathrm{R}$ package "gplots" [53].

\section{Association of IncRNA loci with transcription factor binding sites}

ChIP-seq raw reads for transcription factors p65, IRF3, JunB, cJun and Bcl6 were downloaded from NCBI GEO database (http://www.ncbi.nlm.nih.gov/gds/) (Additional file 11). The 
raw reads were aligned to the mm10 mouse genome build using bowtie 1.0.1 [46] with the $-\mathrm{m}$ reporting option set to 2. The bedgraphs of ChIP-seq were generated using HOMER, where the total number of aligned reads was normalized to 10 million. The peaks of transcription factors were called using MACS [47] following published parameters [48]. The transcription factor binding sites were associated to lncRNA promoter-proximal region $(-10 \mathrm{~kb}$ to $10 \mathrm{~kb}$ from TSS) using BEDtools [42].

\section{Statistical analysis}

Pearson product-moment correlation coefficient was used to measure the linear correlation. Student's t test was used to evaluate the significance of difference for distance to neighboring gene between elncRNAs and plncRNAs. Kolmogorov-Smirnov (K-S) test was performed to evaluate the significance of difference between ecdf curves. All the statistical analyses were performed in $\mathrm{R}$ using the built-in packages.

\section{Additional files}

Additional file 1: Table S1. The NCBI GEO dataset accession numbers for selected microarrays are shown.

Additional file 2: Table S2. The number of microarray probes reannotated for the IncRNAs from Agilent, Illumina and Affymetrix platforms.

Additional file 3: Table S3. The detailed information of IncRNAs identified from Agilent, Illumina and Affymetrix platforms.

Additional file 4: Table S4. The NCBI GEO dataset accession numbers for selected ChIP-seq data are shown.

Additional file 5: Table S5. The list of filtered IncRNAs identified from Agilent, Illumina and Affymetrix platforms.

Additional file 6: Table S6. Reannotation of microarray probes for the protein-coding genes.

Additional file 7: Table S7. The classification of IncRNAs in BMDMs to exonic sense, intronic sense, antisense, bidirectional and intergenic.

Additional file 8: Table S8. The expression profile of IncRNAs in BMDMs integrated from the microarray datasets.

Additional file 9: Figure S1. Correlation between different microarray datasets and integratation of LPS-regulated protein-coding genes from all the datasets. (A) Correlation of the $\log _{2}$ expression change of LPS-regulated protein-coding genes within (upper panel) and across platforms (lower panel). (B) Heatmap of the expression profile of integrated LPS-regulated protein-coding genes across 12 datasets in BMDMs.

Additional file 10: Table S9. The expression profile of protein-coding genes in BMDMs integrated from the microarray datasets.

Additional file 11: Table S10. $q R T-P C R$ validation of an random selection of LPS-regulated IncRNAs.

Additional file 12: Table S11. Significantly changed IncRNAs upon LPS-regulation. P value was derived from robust rank algorithm. Bonferroni method was used to adjust $p$ value. Adjusted $p$ value 0.05 was used as the cutoff to derive the significantly changed IncRNAs. TSS evidence was also shown.

Additional file 13: Table S12. Annotating IncRNAs by chromatin signatures, neighboring protein-coding genes and transcription factors binding sites.
Additional file 14: Table S13. The integrated expression change of LPS-regulated IncRNAs and their closest neighboring genes. LPS-regulated IncRNAs are renamed according to the nearest neighboring protein-coding genes.

Additional file 15: Figure S2. The co-regulation of IncRNA and local mRNA gene pairs stimulated by $10 \mathrm{ng} / \mathrm{ml}, 100 \mathrm{ng} / \mathrm{ml}$ and $1 \mathrm{ug} / \mathrm{ml}$ LPS for 0,3 and 6 hours in BMDMs.

Additional file 16: Table S14. The list of qPCR primers used.

\section{Competing interests}

The authors declare that they have no competing interests.

\section{Authors' contributions}

Conceived and designed the experiments: APM ZZ JS. Performed the experiments: APM JS. Analyzed the data: ZZ APM. Wrote the paper: ZZ APM All authors read and approved the final manuscript.

\section{Acknowledgements}

This work was supported by grants from the National Science Foundation of China (No. 81170362 and No. 81370508).

\section{Author details}

${ }^{1}$ Department of Pathology, Committee on Immunology, University of Chicago, Chicago, Illinois, the United States. ${ }^{2}$ Department of Gastroenterology, Renji Hospital, Shanghai Jiao-Tong University, School of Medicine, Shanghai Institute of Digestive Disease, Shanghai, China. ${ }^{3}$ Department of Medicine, University of Chicago, 900 East 57th street, Chicago, IL 60637, USA.

Received: 26 July 2014 Accepted: 22 January 2015

Published online: 05 February 2015

\section{References}

1. Kawai T, Akira S. The role of pattern-recognition receptors in innate immunity: update on Toll-like receptors. Nat Immunol. 2010;11:373-84.

2. Kung JTY, Colognori D, Lee JT. Long noncoding RNAs: past, present, and future. Genetics. 2013;193:651-69.

3. Cabili MN, Trapnell C, Goff L, Koziol M, Tazon-Vega B, Regev A, et al. Integrative annotation of human large intergenic noncoding RNAs reveals global properties and specific subclasses. Genes Dev. 2011;25:1915-27.

4. Rinn JL, Chang HY. Genome regulation by long noncoding RNAs. Annu Rev Biochem. 2012;81:145-66.

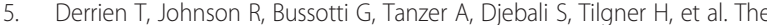
GENCODE v7 catalog of human long noncoding RNAs: analysis of their gene structure, evolution, and expression. Genome Res. 2012;22:1775-89.

6. Fitzgerald KA, Caffrey DR. Long noncoding RNAs in innate and adaptive immunity. Curr Opin Immunol. 2014;26:140-6.

7. Guttman M, Amit I, Garber M, French C, Lin MF, Feldser D, et al. Chromatin signature reveals over a thousand highly conserved large non-coding RNAs in mammals. Nature. 2009:458:223-7.

8. Dave RK, Dinger ME, Andrew M, Askarian-Amiri M, Hume DA, Kellie S. Regulated expression of PTPRJ/CD148 and an antisense long noncoding RNA in macrophages by proinflammatory stimuli. PLOS ONE. 2013;8:e68306.

9. Carpenter S, Aiello D, Atianand MK, Ricci EP, Gandhi P, Hall LL, et al. A long noncoding RNA mediates both activation and repression of immune response genes. Science. 2013;341:789-92.

10. Li Z, Chao T-C, Chang K-Y, Lin N, Patil VS, Shimizu C, et al. The long noncoding RNA THRIL regulates TNFa expression through its interaction with hnRNPL. Proc Natl Acad Sci USA. 2014;111:1002-7.

11. Garmire LX, Garmire DG, Huang W, Yao J, Glass CK, Subramaniam S. A global clustering algorithm to identify long intergenic non-coding RNA-with applications in mouse macrophages. PLoS ONE. 2011;6:e24051.

12. Ilott NE, Heward JA, Roux B, Tsitsiou E, Fenwick PS, Lenzi L, et al. Long non-coding RNAs and enhancer RNAs regulate the lipopolysaccharide-induced inflammatory response in human monocytes. Nat Commun. 2014:5:3979.

13. Du Z, Fei T, Verhaak RGW, Su Z, Zhang Y, Brown M, et al. Integrative genomic analyses reveal clinically relevant long noncoding RNAs in human cancer. Nat Struct Mol Biol. 2013;20:908-13. 
14. Gellert P, Ponomareva Y, Braun T, Uchida S. Noncoder: a web interface for exon array-based detection of long non-coding RNAs. Nucleic Acids Res. 2013;41:e20

15. Liao Q, Xiao H, Bu D, Xie C, Miao R, Luo H, et al. ncFANs: a web server for functional annotation of long non-coding RNAs. Nucleic Acids Res. 2011:39(Web Server issue):W118-24.

16. Mercer TR, Dinger ME, Sunkin SM, Mehler MF, Mattick JS. Specific expression of long noncoding RNAs in the mouse brain. Proc Natl Acad Sci USA. 2008;105:716-21.

17. Liao Q, Liu C, Yuan X, Kang S, Miao R, Xiao H, et al. Large-scale prediction of long non-coding RNA functions in a coding-non-coding gene co-expression network. Nucleic Acids Res. 2011;39:3864-78.

18. Jia H, Osak M, Bogu GK, Stanton LW, Johnson R, Lipovich L. Genome-wide computational identification and manual annotation of human long noncoding RNA genes. RNA. 2010;16:1478-87.

19. Zhang X, Sun S, Pu JKS, Tsang ACO, Lee D, Man VOY, et al. Long non-coding RNA expression profiles predict clinical phenotypes in glioma. Neurobiol Dis. 2012;48:1-8.

20. Raghavachari N, Barb J, Yang Y, Liu P, Woodhouse K, Levy D, et al. A systematic comparison and evaluation of high density exon arrays and RNA-seq technology used to unravel the peripheral blood transcriptome of sickle cell disease. BMC Med Genomics. 2012;5:28.

21. Xu W, Seok J, Mindrinos MN, Schweitzer AC, Jiang H, Wilhelmy J, et al. Human transcriptome array for high-throughput clinical studies. Proc Natl Acad Sci USA. 2011;108:3707-12.

22. Shiraki T, Kondo S, Katayama S, Waki K, Kasukawa T, Kawaji H, et al. Cap analysis gene expression for high-throughput analysis of transcriptional starting point and identification of promoter usage. Proc Natl Acad Sci USA. 2003:100:15776-81.

23. Plessy $\mathrm{C}$, Bertin $\mathrm{N}$, Takahashi $H$, Simone $\mathrm{R}$, Salimullah $\mathrm{M}$, Lassmann $\mathrm{T}$, et al. Linking promoters to functional transcripts in small samples with nanoCAGE and CAGEscan. Nat Methods. 2010;7:528-34.

24. Pauli A, Valen E, Lin MF, Garber M, Vastenhouw NL, Levin JZ, et al. Systematic identification of long noncoding RNAs expressed during zebrafish embryogenesis. Genome Res. 2012;22:577-91.

25. Kolde R, Laur S, Adler P, Vilo J. Robust rank aggregation for gene list integration and meta-analysis. Bioinformatics. 2012;28:573-80.

26. Alvarez-Dominguez JR, Hu W, Yuan B, Shi J, Park SS, Gromatzky AA, et al. Global discovery of erythroid long noncoding RNAs reveals novel regulators of red cell maturation. Blood. 2014;123:570-81.

27. Marques AC, Hughes J, Graham B, Kowalczyk MS, Higgs DR, Ponting CP. Chromatin signatures at transcriptional start sites separate two equally populated yet distinct classes of intergenic long noncoding RNAs. Genome Biol. 2013;14:R131.

28. Ørom UA, Shiekhattar R. Long non-coding RNAs and enhancers. Curr Opin Genet Dev. 2011;21:194-8.

29. Natoli G, Andrau J-C. Noncoding transcription at enhancers: general principles and functional models. Annu Rev Genet. 2012;46:1-19.

30. De Santa F, Barozzi I, Mietton F, Ghisletti S, Polletti S, Tusi BK, et al. A large fraction of extragenic RNA pol II transcription sites overlap enhancers. PLoS Biol. 2010:8:e1000384.

31. Wang KC, Yang YW, Liu B, Sanyal A, Corces-Zimmerman R, Chen Y, et al. A long noncoding RNA maintains active chromatin to coordinate homeotic gene expression. Nature. 2011;472:120-4.

32. Hu G, Tang Q, Sharma S, Yu F, Escobar TM, Muljo SA, et al. Expression and regulation of intergenic long noncoding RNAs during T cell development and differentiation. Nat Immunol. 2013;14:1190-8.

33. Barish GD, Yu RT, Karunasiri M, Ocampo CB, Dixon J, Benner C, et al. Bcl-6 and NF-kappaB cistromes mediate opposing regulation of the innate immune response. Genes Dev. 2010;24:2760-5.

34. Ellertsen LK, Hetland G, Johnson E, Grinde B. Effect of a medicinal extract from Agaricus blazei Murill on gene expression in a human monocyte cell line as examined by microarrays and immuno assays. Int Immunopharmacol. 2006;6:133-43.

35. Melgar MF, Collins FS, Sethupathy P. Discovery of active enhancers through bidirectional expression of short transcripts. Genome Biol. 2011:12:R113.

36. Kim T-K, Hemberg M, Gray JM, Costa AM, Bear DM, Wu J, et al. Widespread transcription at neuronal activity-regulated enhancers. Nature. 2010:465:182-7.

37. Pruitt KD, Tatusova T, Brown GR, Maglott DR. NCBI Reference Sequences (RefSeq): current status, new features and genome annotation policy. Nucleic Acids Res. 2012;40(Database issue):D130-5
38. Dreszer TR, Karolchik D, Zweig AS, Hinrichs AS, Raney BJ, Kuhn RM, et al. The UCSC Genome Browser database: extensions and updates 2011 Nucleic Acids Res. 2012;40(Database issue):D918-23.

39. Carninci P, Kasukawa T, Katayama S, Gough J, Frith MC, Maeda N, et al. The transcriptional landscape of the mammalian genome. Science. 2005;309:1559-63.

40. Flicek P, Amode MR, Barrell D, Beal K, Billis K, Brent S, et al. Ensembl 2014 Nucleic Acids Res. 2014;42(Database issue):D749-55.

41. Kent WJ. BLAT-the BLAST-like alignment tool. Genome Res. 2002;12:656-64.

42. Quinlan AR, Hall IM. BEDTools: a flexible suite of utilities for comparing genomic features. Bioinformatics. 2010;26:841-2.

43. Yamashita R, Sugano S, Suzuki Y, Nakai K. DBTSS: DataBase of Transcriptional Start Sites progress report in 2012. Nucleic Acids Res. 2012:40(Database issue):D150-4

44. Shumway M, Cochrane G, Sugawara H. Archiving next generation sequencing data. Nucleic Acids Res. 2010:38(Database issue):D870-1.

45. Li H, Durbin R. Fast and accurate short read alignment with Burrows-Wheeler transform. Bioinformatics. 2009;25:1754-60.

46. Langmead B, Trapnell C, Pop M, Salzberg SL. Ultrafast and memory-efficient alignment of short DNA sequences to the human genome. Genome Biol. 2009;10:R25

47. Zhang Y, Liu T, Meyer CA, Eeckhoute J, Johnson DS, Bernstein BE, et al. Model-based analysis of ChIP-Seq (MACS). Genome Biol. 2008;9:R137.

48. Feng J, Liu T, Zhang Y. Using MACS to identify peaks from ChIP-Seq data. Curr Protoc Bioinformatics. 2011; Chapter 2:Unit 2.14.

49. Barrett T, Wilhite SE, Ledoux P, Evangelista C, Kim IF, Tomashevsky M, et al. NCBI GEO: archive for functional genomics data sets-update. Nucleic Acids Res. 2013;41(Database issue):D991-5.

50. Lopez-Romero P. Agi4x44PreProcess. R package version 1.16.0, http://www. bioconductor.org/packages//2.10/bioc/html/Agi4x44PreProcess.html.

51. Venables WN, Ripley BD. Modern Applied Statistics with S. Fourthth ed New York: Springer; 2002. ISBN 0-387-95457-0.

52. Morgan $M$, Pagès $H$, Obenchain $V$ and Hayden $N$. Rsamtools: Binary alignment (BAM), FASTA, variant call (BCF), and tabix file import. $R$ package version 1.18.2, http://bioconductor.org/packages/release/bioc/html/ Rsamtools.html.

53. Warnes GR, Bolker B, Bonebakker L, Gentleman R, Huber W, et al. (2012) Package 'gplots'. http://cran.r-project.org.

\section{Submit your next manuscript to BioMed Central and take full advantage of:}

- Convenient online submission

- Thorough peer review

- No space constraints or color figure charges

- Immediate publication on acceptance

- Inclusion in PubMed, CAS, Scopus and Google Scholar

- Research which is freely available for redistribution 